\title{
For an instant WOW effect
}

Dr Michaela Sehnert, a general dentist from Leipzig, Germany with a special interest in clear

aligner therapy in combination with other treatments for fast, predictable and minimal- and

non-invasive solutions, explains why she went digital three years ago.

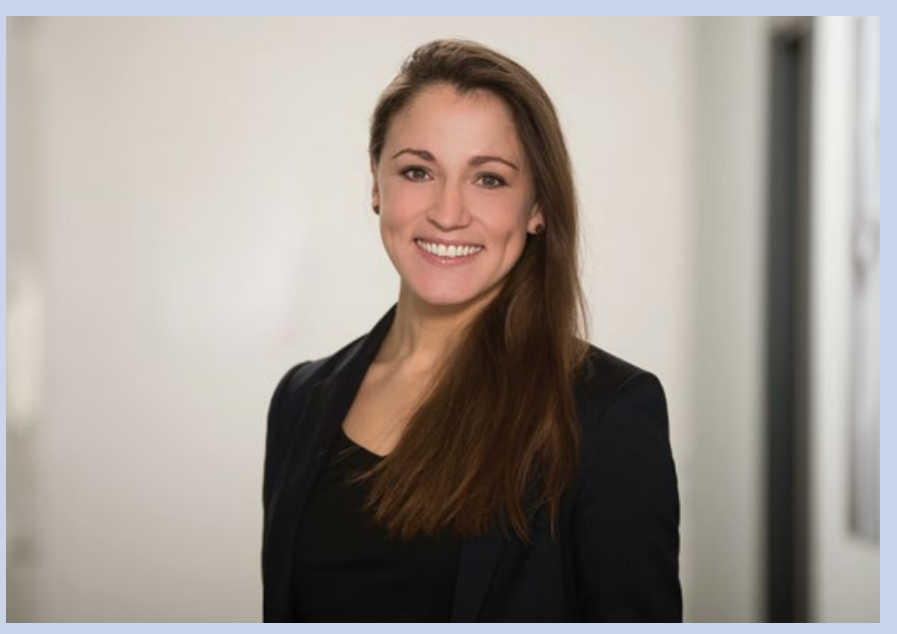

Dr Michaela Sehnert

The main decision to make the practice fully digital was initially about time saving. However, I found I was then able to delegate a greater amount of work to my staff. Going fully digital also allowed me to remain completely up to date with all of my customers, as well as with my laboratory partners. Sending and receiving files could be done without wasting time. No-one needed to collect or drop off impressions anymore; instead, all we needed to do was upload pictures and scans, communicate with the technician in the space of approximately two minutes - and all that without having to move from our locations both the practice and the lab. Plus, we could then immediately share treatment plans whilst the patient was still in the dental chair.

In the first instance we are able to show the patient a treatment outcome before they embark on treatment - and fast. We scan using the iTero digital scanner and in approximately two minutes we have an outcome to share with the patient which we can then use to explain what we hope to achieve ... and how. Even at this early stage we can say that with the Invisalign treatment, and a restorative phase, this is what we hope to achieve.

As a next step we are then able to match the patient's photos and digital scan together and compare the look of their current dentition, with the outcome shown on the ClinCheck - Align's proprietary software. We show a predictable result on a $3 \mathrm{D}$ model and can tell the patient what the treatment time is going to be to reach this result.

With the Invisalign Outcome Simulator, you get an instant WOW effect and that's exactly what you need to convince patients to consider starting treatment. You can show them what we can do with their teeth in the space of a few minutes. My team are able to do all this by themselves; they get all the emotional feedback from patients who are blown away by what we can do for them. Predictability is so powerful, and the most important criterion in helping them reach a decision is what they see before and what they will get. With the
Invisalign workflow, you get a clear idea of the number of aligners you need, how many appointments need to be scheduled and at what intervals, how long the treatment is going to be.

And as long as the aligners fit, we are all ok. The first step in the process is that Align Technology sends us the treatment plan, so even before the patients get their aligners, we know how many aligners we will be giving the patient, when they need to change aligners and subsequently when we need to schedule each appointment. And the predictability means that you can stick to the treatment plan $99 \%$ of the time. Patients are also able to see that everything is on track, they are able to check their Invisalign mobile app and see for themselves what is going on with their teeth.

The iTero Element 5D and ClinCheck software can help us decide which treatment the patient needs, how minimally invasive it can be, when it needs to be performed, and which treatment is going to be carried out first in my complete treatment plan.

The Invisalign system is definitely suitable for comprehensive dentistry; sometimes we are lucky enough to have a straightforward orthodontic case, then we may do a bit of bleaching and composite bonding or fit some veneers. But sometimes we need iTero or the ClinCheck to understand how to plan a more complex restorative case. It may be that I need to create space or fill space, look at whether we can plan a minimally invasive treatment or what additional treatments we actually need to restore a patient's mouth, and at what stage. Using the Invisalign/iTero system as part of this is a perfect way to give us that predictability needed to plan implant treatments. And actually, it is of huge benefit for my patients too, as the cost estimation is a lot more realistic because we know what we are planning before we even start.

Some patients feel that their treatment is progressing slowly. So we have the functionality on the scanner which shows them what progress has been achieved visually from one appointment to the next. It also indicates if the treatment is proceeding as planned. It is all about keeping patients engaged whilst using time effectively.

Our treatment outcomes can be made more predictable and these can help us make the right decisions too. You are given all the tools to figure out at which point a specific treatment is best carried out; do I do my Invisalign treatment first, and then place my implants?

The iTero Element 5D and ClinCheck software can help us decide which treatment the patient needs, how minimally invasive it can be, when it needs to be performed, and which treatment should be carried out first in my complete treatment plan.

Thanks to the iTero and ClinCheck, my time is used most efficiently, which is money saving for us and actually money saving for the patient too.

For more information about how an iTero intraoral scanner could transform your practice, please visit www.iTero.com, or contact your local Invisalign or iTero representative. 